\title{
MBEP
}

\section{Profesores de Educación: perfiles y prácticas profesionales}

María Eugenia Vicente

http://dx.doi.org/10.1590/S2176-6681/313512567

\section{Resumen}

En el marco de los cambios ocurridos en el campo profesional de las Ciencias de la Educación, el presente artículo es un trabajo de investigación cuyo objetivo es reconstruir los perfiles de los egresados en relación con: la edad, el género, la formación, la constitución familiar, el trabajo y los lugares de residencia. Para ello, se utilizan tres fuentes de datos, 730 legajos de los egresados, un cuestionario aplicado a una muestra de 92 egresados y censos o anuarios estadísticos que permiten contextualizar la unidad de análisis. Las características del perfil sociodemográfico de los egresados en Ciencias de la Educación aportan datos para avanzar con futuras investigaciones en relación a las biografías y trayectorias laborales y educativas de los egresados.

Palabras clave: Ciencias de la Educación; perfil sociodemográfico; egresados; campo profesional. 


\section{Abstract \\ Education Teachers: profiles and professional practices}

As part of the changes in the professional field of Education Sciences, this article is a research project, which aims to reconstruct the profiles of graduates in relation to: age, gender, education, family constitution, work and places of residence. To do this, we use three data sources, files of 730 graduates, a questionnaire administered to a sample of 92 graduates and censuses or statistical yearbooks, which allow the contextualization of the unit of analysis. The characteristics of social and demographic profile of graduates in Education Sciences provide data to advance future researches in relation to the biographies, work and educational paths of graduates.

Keywords: Education Sciences; demographic profile; graduates; professional field.

\section{Resumo \\ Professores de Educação: perfis e práticas profissionais}

Perante as mudanças ocorridas no campo profissional de Ciências da Educação, este artigo é um projeto de pesquisa cujo objetivo é reconstruir os perfis dos formandos em relação a: idade, gênero, formação, constituição familiar, trabalho e locais de residência. Para isso, usamos três fontes de dados, 730 arquivos de licenciados, um questionário aplicado a uma amostra de 92 formandos e censos ou anuários estatísticos que permitem contextualizar a unidade de análise. As características do perfil sociodemográfico dos licenciados em Ciências da Educação fornecem dados para avançar a pesquisa futura em relação às biografias e aos percursos educativos e de trabalho de graduados.

Palavras-chave: Ciências da Educação; perfil demográfico; graduados; campo profissional.

\section{Introducción}

Durante los últimos tiempos, los campos profesionales han sufrido intensas modificaciones y las Ciencias de la Educación no estuvieron exentas. En primera instancia, el trabajo se presenta más como una experiencia compleja que como el cumplimiento de un rol. Al respecto, Dubet $(2006,2007)$ señala que durante mucho tiempo el trabajo sobre los otros procedía de un programa institucional y designaba un modo de socialización, un tipo de relación con el otro. Según este programa, el trabajo sobre el otro era una mediación entre los valores universales y 
los individuos particulares; el trabajo de socialización era una vocación porque se encontraba directamente fundado sobre valores; y que la socialización está orientada a inculcar normas que configuran al individuo y simultáneamente lo vuelven autónomo y libre.

No obstante, Dubet $(2006,2007)$ reconoce que en la actualidad existe una descomposición de elementos y representaciones que el programa institucional tuvo la capacidad de integrar en un sistema percibido como más o menos coherente. Los "valores" perdieron su unidad; la "vocación" choca contra los requerimientos de eficacia profesional, contra los constreñimientos de organizaciones más lábiles y más complejas, y la creencia en una continuidad entre socialización y subjetivación ya no resulta evidente.

En segunda instancia, han cambiado las condiciones objetivas tanto del diagnóstico como de la solución de los problemas básicos. Al respecto, Tenti Fanfani y Campo (1990) señalan que el ejercicio profesional se inscribe dentro de espacios sociales más complejos, lo cual obliga a interactuar con colegas, con otros especialistas, con administradores y con clientes colectivos cuyos problemas no aparecen delimitados en forma clara e inmediata. De esta manera, esta complejidad genera una serie de tendencias hacia la diferenciación de las profesiones en especialidades y sub-especialidades. Esto acrecienta la disputa por el problema de las jurisdicciones entre profesiones y sus respectivas especialidades, los cuales mantienen relaciones de competencia para la realización de tareas específicas. En este sentido, Fernández (1989) y Furlán (1989) afirman que las Ciencias de la Educación como profesión se encuentran en encrucijada; las luchas y disputas interprofesionales por la dominación y posesión del campo profesional han tenido un carácter permanente.

Para el caso particular de las Ciencias de la Educación, en efecto, los sujetos y contextos de ejercicio profesional han cambiado. En los orígenes de este campo, en 1914, los docentes eran los destinatarios principales de las prácticas profesionales de los egresados, la práctica de formación docente y el espacio del sistema educativo contribuyeron a la institucionalización de las Ciencias de la Educación. En la actualidad, el campo profesional se constituye de múltiples referencias socioprofesionales por fuera del sistema educativo y de la profesión docente, teniendo como destinatarios no sólo a docentes y escolares, pero también a los trabajadores, a los ciudadanos en general, a los visitantes de museos, a los artistas, a los empresarios, a los adultos mayores, a los pacientes de hospitales. En este sentido, este trabajo de investigación pretende aportar características y orientaciones de la estructura del campo profesional de las Ciencias de la Educación y donde anclar posteriores estudios sobre las prácticas, experiencias y espacios que el análisis cualitativo pueda abordar en su complejidad y diversidad.

\section{Metodología}

Este trabajo forma parte de una tesis de doctorado cuyo objetivo es analizar las trayectorias profesionales de los egresados en Ciencias de la 
Educación de la Universidad Nacional de La Plata (UNLP), en Argentina. El trabajo de investigación aquí presentado describe el perfil socioprofesional, familiar y educativo de los egresados en Ciencias de la Educación entre los años 1970 a 2009 de la UNLP.

Como herramienta de recolección de datos se utiliza un cuestionario diseñado en torno de cinco ejes: I) datos generales (año de graduación, lugar de residencia y procedencia); II) estudios; III) situación familiar; IV) consumos/preferencias culturales y V) trabajo.

En relación a la población objeto de estudio (Cuadro 1), son 878 egresados en Ciencias de la Educación de la Universidad Nacional de La Plata. Dicha institución fue elegida por un criterio fundacional, es allí donde se abre la carrera por primera vez en Argentina. Respecto del recorte temporal, la población corresponde a quienes egresaron entre los años 1970 a 2009 debido a que, por un lado, analizar el campo profesional a partir de la década de 1970 ofrece un panorama mayor de tiempo y permite captar los cambios acontecidos en el campo profesional a partir de la década del 80 (como se verá más adelante). Por otro lado, el recorte finaliza en el año 2009 para lograr aportar conocimiento sobre el campo y los actores en la actualidad.

La muestra utilizada para el cuestionario es de noventa y dos (92) egresados, correspondiente al 10\% aproximadamente del total de egresados por cada década. Inicialmente, se contactaron a 150 graduados de los que se logró conseguir los datos de contacto (mail, teléfono, redes sociales), de los cuales aproximadamente 110 egresados contestaron que aceptaban contestar la encuesta y algunos de éstos proporcionaron datos de contactos de otros egresados que se sumaron. Quienes finalmente aceptaron y contestaron la encuesta a febrero de 2013 son 92 egresados, la muestra final.

\section{Cuadro 1 - Muestra de Egresados en Ciencias de la Educación de la UNLP entre los Años 1970 a 2009 con la que se Trabajó en esta Tesis en Relación a la Población de Estudio}

\begin{tabular}{|l|r|r|r|r|r|}
\hline Década & 1970-1979 & 1980-1989 & 1990-1999 & 2000-2009 & Totales \\
\hline Población & 251 & 273 & 206 & 229 & 959 \\
\hline Muestra & 13 & 28 & 27 & 24 & 92 \\
\hline$\%$ & 6 & 10 & 13 & 10 & 10 \\
\hline
\end{tabular}

Fuente: elaboración propia

Finalmente, el análisis de los datos, de tipo estadístico, se orientó a reconstruir el perfil socioprofesional del conjunto de los egresados, a partir de los siguientes indicadores: edad, género, constitución familiar, las prácticas profesionales y los lugares de residencia.

\section{Resultados}

\section{Características generales}

Según el Cuadro 2, respecto de la población universitaria de las universidades en Argentina tanto públicas como privadas, entre los años 
1995 y 1999, ingresaron 1.451.622, egresaron 231.511 y 5.219 .098 eran estudiantes. Por su parte, entre los años 2000 y 2004, ingresaron 1.797.968, egresaron 365.653 y 7.240 .994 eran estudiantes. Finalmente, entre los años 2005 y 2009, ingresaron 1.835.931, egresaron 451.230, y 7.959 .957 eran estudiantes. En términos relativos, las proporciones de los índices de cada grupo universitario presentan ciertas diferencias de crecimiento. Esto es, de la población estudiantil total de las universidades argentinas públicas y privadas, entre 1995 y 1999, 76\% eran estudiantes, 21\% ingresantes y 3\% egresados; entre los años 2000 y 2004, 77\% eran estudiantes, 19\% eran ingresantes y $4 \%$ eran egresados. Por su parte, entre los años 2005 a 2009, $78 \%$ eran estudiantes, $17 \%$ eran ingresantes y $5 \%$ eran egresados. Esto significa que desde 1995 a 2009 hubo un aumento de la cantidad de egresados universitarios: de 3\% de la población total universitaria en 1995 a 5\% en 2009; también aumentó la cantidad de estudiantes, aquella parte de la población universitaria que permanece en el nivel, que en 1995 conformaban el 76\% del total y para el año 2009 constituían el 78\%. Pero el índice de ingresantes no presenta el mismo crecimiento, más bien, decreció la cantidad de ingresantes a las universidades argentinas, de 21\% que conformaban el total de la población universitaria en 1995 a 17\% en el año 2009.

\section{Cuadro 2 - Distribución de los Estudiantes, Inscriptos y Egresados de Universidades Públicas y Privadas entre 1995 y 2009, cada Cuatro Años}

\begin{tabular}{|l|c|c|c|}
\hline \multicolumn{1}{|c|}{ Universidades en total: } & $1995-1999$ & $2000-2004$ & $2005-2009$ \\
\hline Estudiantes & 5.219 .098 & 7.240 .994 & 7.959 .957 \\
\hline Inscriptos & 1.451 .622 & 1.797 .968 & 1.835 .931 \\
\hline Egresados & 231.511 & 365.653 & 451.230 \\
\hline
\end{tabular}

Fuente: elaboración propia en base a datos de la Secretaría de Políticas Universitarias. Ministerio de Educación de la Nación.

En el marco de los índices y características de crecimiento de la población universitaria argentina, en 2004 la carrera de Ciencias de la Educación se ubicaba en el noveno lugar de las veinte carreras universitarias más elegidas después de Abogacía, Contador Público, Psicología, Administración, Sistemas, Medicina, Comunicación Social y Enfermería (SPU, 2004). En relación a la evolución de la matrícula de estudiantes y los índices de ingreso y egreso de las diferentes carreras de Ciencias de la Educación de la Argentina, según el Cuadro 3, aumentó la cantidad de estudiantes. Entre 1982 y 1990 correspondían al 74\% de la cantidad de universitarios en la carrera, y para los años 2001 a 2009 corresponden al $76 \%$. También aumentó la cantidad de egresados de las carreras de Ciencias de la Educación, de 3\% que representaban en el total de los universitarios en la carrera entre los años 1982 a 1990, a 4\% entre los años 2000 a 2009. 
Por su parte, disminuyó la cantidad de ingresantes a las carreras de Ciencias de la Educación en Argentina, entre 1982 y 1990 representaban el 23\%, mientras que entre los años 2000 a 2009 representan el 20\%.

El comportamiento de los índices de egresos, ingresos y la matrícula de estudiantes de todas las carreras de Ciencias de la Educación de Argentina corresponden a los ritmos de crecimiento de la población universitaria en general. En este sentido, la disminución de la cantidad de ingresantes a Ciencias de la Educación no implica que haya menos estudiantes que elijan la carrera, sino que refleja un fenómeno presente en el nivel en general, puesto que en las últimas dos décadas hubo una disminución de la cantidad de ingresantes al sistema universitario.

\section{Cuadro 3 - Distribución de los Estudiantes, Inscriptos y Egresados de Ciencias de la Educación de Universidades Públicas y Privadas, de Pregrado, Grado y Posgrado, entre 1982 y 2009}

\begin{tabular}{|l|c|c|c|}
\hline \multicolumn{1}{|c|}{ Ciencias de la Educación: } & 1982-1990 & 1991-1999 & 2001-2009 \\
\hline Estudiantes & 59.601 & 105.046 & 192.090 \\
\hline Inscriptos & 18.317 & 34.303 & 51.002 \\
\hline Egresados & 2.557 & 4.633 & 8.192 \\
\hline
\end{tabular}

Fuente: elaboración propia en base a datos de la Secretaría de Políticas Universitarias. Ministerio de Educación de la Nación.

Como se mencionó anteriormente, la carrera de Ciencias de la Educación se ubica entre las carreras más elegidas del país. En relación a los datos de los Cuadros 2 y 3, en la última década la carrera tuvo 8.192 egresados que formaban parte de los 816.883 egresados en total de todas las universidades argentinas. Ingresaron a la carrera 51.002 nuevos inscriptos que forman parte de los 3.633.899 ingresantes al sistema universitario en total; y la carrera tenía la cantidad de 192.090 estudiantes que, a su vez, formaban parte de los 15.200.951 estudiantes en el nivel universitario.

En este marco, y acorde a lo presentado en el Cuadro 4, la carrera de Ciencias de la Educación se ofrece en 29 universidades en Argentina, de las cuales 16 son universidades estatales y 13 son privadas. La mayoría ubicadas en la región del Centro con 16 universidades, 14 estatales y 12 privadas; en menor medida se ubica la región del noreste, con 6 universidades que ofrecen la carrera, de las cuales 5 son estatales y 1 privada; en la región de Cuyo se encuentran 3 universidades estatales que ofrecen la carrera; en la región patagónica también son 3 las universidades estatales que ofrecen Ciencias de la Educación y finalmente se encuentra 1 universidad estatal en la región del noroeste que dicta la carrera. En este mapa de la oferta de formación en Ciencias de la Educación, la región del centro concentra las universidades privadas del país que ofrecen la carrera, mientras que en la mayoría de las demás regiones la carrera es ofrecida por universidades estatales. 


\section{Cuadro 4 - Distribución de las Universidades Públicas y Privadas que Ofrecen la Carrera de Ciencias de la Educación, según Región ${ }^{1}$, al 2013}

\begin{tabular}{|l|c|c|c|c|c|c|}
\hline & \multicolumn{2}{|c|}{$\begin{array}{c}\text { Cantidad de universidades que } \\
\text { ofrecen la carrera }\end{array}$} & \multicolumn{3}{c|}{ Cantidad de carreras de } \\
\hline Región & Públicas & Privadas & Total & $\begin{array}{c}\text { Profesorado } \\
\text { en Ciencias de } \\
\text { la Educación }\end{array}$ & $\begin{array}{c}\text { Licenciatura } \\
\text { en Ciencias de } \\
\text { la Educación }\end{array}$ & Total \\
\hline Noroeste & 5 & 1 & 6 & 5 & 5 & 10 \\
\hline Noreste & 1 & 0 & 1 & 1 & 1 & 2 \\
\hline Cuyo & 3 & 0 & 3 & 3 & 3 & 6 \\
\hline Centro & 14 & 12 & 16 & 19 & 27 & 46 \\
\hline Patagonia & 3 & 0 & 3 & 3 & 2 & 5 \\
\hline
\end{tabular}

Fuente: elaboración propia en base a datos de la Secretaría de Políticas Universitarias. Ministerio de Educación de la Nación.

En síntesis, respecto de los egresados de la carrera de Ciencias de la Educación de la Universidad Nacional de La Plata, objeto de análisis del presente artículo, y como se observa en los Cuadros 1 y 3 , entre los años 1970 a 1979 egresaron 251 estudiantes; entre los años 1980 a 1989 egresaron 273 estudiantes, que forman parte de los 2.557 egresados en Ciencias de la Educación en Argentina para el mismo período; entre los años 1990 a 1999 egresaron 206 estudiantes, que forman parte de los 4.633 egresados de la carrera en Argentina para el mismo período; finalmente, entre los años 2000 a 2009, egresaron 229 estudiantes de Ciencias de la Educación de la Universidad Nacional de La Plata, que forman parte de los 8.192 egresados en total de dicha carrera, durante la última década.

\section{La formación}

Las instituciones educativas por las que transitaron los egresados en Ciencias de la Educación podrían constituir ciertos circuitos educativos que explicarían el perfil de los egresados. El Cuadro 5 presenta datos sobre las escuelas secundarias a las que asistieron los egresados en función del sector y del tipo de institución.

Como se observa en el Cuadro 5, el 63\% de los egresados en Ciencias de la Educación asistieron a escuelas públicas, y dentro del circuito público de instituciones educativas, asistieron principalmente a las escuelas normales (29\%). Según Martínez y Southwell (2011), el normalismo constituye uno de los discursos pedagógicos más influyentes dentro del espacio educativo y cultural nacional que, a través de las escuelas normales, se convirtió en una matriz de pensamiento para muchas generaciones de educadores. El normalismo organizó la institucionalización de la pedagogía a través de normar hasta sus actos más cotidianos, considerando la escuela como el espacio privilegiado para la enseñanza de los valores de lo público y posicionando al docente como funcionario, ubicado en el lugar de ser el

\footnotetext{
${ }^{1}$ Las regiones están agrupadas según el criterio del INDEC: Noroeste (Jujuy, Salta, Tucumán, Santiago del Estero, Catamarca y La Rioja), Noreste (Formosa, Chaco, Misiones y Corrientes); Cuyo (San Juan, San Luis y Mendoza); Pampeana (Santa Fe, Entre Ríos, Córdoba, La Pampa y Buenos Aires) y Patagonia (Neuquén, Río Negro, Chubut, Santa Cruz y Tierra del Fuego).
} 
principal dispensador de los valores que estaban configurando el Estadonación que nacía. En los inicios de la carrera de Ciencias de la Educación, según el art. 42 del Reglamento y Plan de Estudios de la Facultad de Ciencias de la Educación, los requisitos de ingreso eran:

Para ingresar en los cursos que se dictan en la Facultad [de Ciencias de la Educación], para las carreras del Profesorado Secundario y el Doctorado se requiere: a) Estudios completos de segunda enseñanza de los Colegios Nacionales de la República, b) Título de Profesor Normal, c) Título de Maestro Normal, d) Título universitario o inscripción en una de las Facultades o Institutos de la universidad" (Archivos de Ciencias de la Educación, 1914).

\section{Cuadro 5 - Distribución de Egresados en Ciencias de la Educación de la UNLP entre los Años 1970 a 2009 según la Formación de Nivel Secundario, en Porcentajes $(\mathrm{N}=92)$}

\begin{tabular}{|c|l|r|}
\hline \multirow{2}{*}{ Sector educativo } & Institución de Educación Secundaria & Cantidad de egresados \\
\hline \multirow{2}{*}{ Público } & Escuelas Normales & 29,34 \\
\hline & Colegios Nacionales & 9,78 \\
& Liceo y Bachillerato Universitario & 10,86 \\
\cline { 2 - 3 } & Escuelas de enseñanza básica & 10,86 \\
& Técnicas y Agrícolas & 2,17 \\
\hline \multirow{2}{*}{ Privado } & - & 29 \\
\hline & Total & 100 \\
\hline
\end{tabular}

Fuente: elaboración propia.

El título de maestro o profesor normal como requisito para ingresar a la Facultad de Ciencias de la Educación fue desapareciendo con las transformaciones de los reglamentos, los planes de estudios de la carrera y las titulaciones otorgadas, tal como se verá en el próximo apartado.

\section{El género, la edad y el magisterio}

Ser maestro y también ser egresado de Ciencias de la Educación lejos de ser un requisito, es una característica de la matrícula de los egresados hasta la actualidad, como lo muestra el Cuadro 6.

La mayoría de los egresados en Ciencias de la Educación han transitado por un circuito de formación docente que comienza en el nivel secundario y avanza en el nivel terciario. No obstante, con el paso del tiempo la cantidad de egresados que poseen título docente decrece y podría deberse a la terciarización del magisterio que hubiese provocado un retraso en la obtención de la certificación y su consecuente acceso al mercado de trabajo. Pogré (2004) apunta que en 1969 se terciariza - por decreto presidencial - la formación de maestros primarios. La nueva carrera magisterial abarcaría dos años y medio de formación y tendría como requerimiento de ingreso poseer certificado de aprobación del nivel medio. 


\begin{abstract}
Cuadro 6 - Distribución de la Población de Egresados entre los Años 1970 a 1995 según edad al Momento del Egreso y Posesión de Título Docente Previo al Ingreso a Ciencias de la Educación, en Números Absolutos $(\mathrm{N}=668)$
\end{abstract}

\begin{tabular}{|c|c|c|c|c|c|c|}
\hline & \multicolumn{2}{|c|}{ 1970-1979 } & \multicolumn{2}{|c|}{ 1980-1989 } & \multicolumn{2}{|c|}{ 1991-1995 } \\
\hline & $\begin{array}{c}\text { Total de } \\
\text { egresados }\end{array}$ & $\begin{array}{l}\text { Docentes } \\
\text { sobre el } \\
\text { total de } \\
\text { egresados }\end{array}$ & $\begin{array}{c}\text { Total de } \\
\text { egresados }\end{array}$ & $\begin{array}{l}\text { Docentes } \\
\text { sobre el } \\
\text { total de } \\
\text { egresados }\end{array}$ & $\begin{array}{c}\text { Total de } \\
\text { egresados }\end{array}$ & $\begin{array}{c}\text { Docentes } \\
\text { sobre el } \\
\text { total de } \\
\text { egresados }\end{array}$ \\
\hline Menor de 25 & 111 & 83 & 158 & 18 & 83 & 8 \\
\hline Entre 25-30 & 100 & 80 & 80 & 7 & 43 & 1 \\
\hline Entre 31-35 & 15 & 12 & 20 & 8 & 11 & 1 \\
\hline Mayor de 36 & 25 & 23 & 15 & 13 & 7 & 4 \\
\hline Totales & 251 & 198 & 273 & 46 & 144 & 14 \\
\hline
\end{tabular}

Fuente: elaboración propia en base a legajos egresados Ciencias de la Educación.

En el marco del modelo tecnicista se introdujo una división técnica del trabajo escolar separando los planificadores, los evaluadores, los supervisores y los docentes que eran concebidos como técnicos, quienes bajaban a la práctica, de manera simplificada, el currículum prescripto alrededor de objetivos de conducta y medición de rendimientos. Progresivamente se incorporaron diversas instituciones superiores a la oferta de formación docente: escuelas normales, institutos nacionales de profesorado, institutos provinciales, instituciones superiores de formación técnica y universidades, tanto oficiales como privadas. A partir de 1980, aquellos que obtenían un título de nivel terciario, directamente se insertarían en el mercado laboral sin proseguir estudios universitarios de Ciencias de la Educación o a la inversa.

Con respecto a la caracterización de los egresados en relación al género, según el Cuadro 7, la carrera se presenta altamente feminizada. La carrera en sus orígenes se constituyó para la formación de quienes enseñarían en las aulas de las instituciones educativas. Según el primer Plan de Estudios de la Facultad de Ciencias de la Educación de la UNLP (1914), se otorgaban los títulos de Profesor de Enseñanza Primaria, Profesor de enseñanza Secundaria, Normal y Especial, Profesor de Enseñanza Especial en dibujo y Música, Doctor en Ciencias de la Educación. El significado social otorgado a la docencia se constituía a partir de relacionar las imágenes de la maestra como segunda madre y la escuela como segundo hogar. Ser madre y ser esposa era el único destino de la mujer, el cual, aunado con el de profesora, quedaba como el lugar propio de la mujer, en el que la enseñanza se definía como una extensión del trabajo productivo y reproductivo que las mujeres realizaban en el hogar (Echeverría, 2002).

La carrera de Ciencias de la Educación está altamente feminizada, el 91\% de la muestra analizada son mujeres, constituyéndose en heredera de la tradición aquella que une la figura de la mujer con la formación de los ciudadanos. Pero al mismo tiempo forma parte de un fenómeno social mayor 
ya que la Argentina tiene la fuerza del trabajo docente más feminizada, en comparación con Brasil, Perú y Uruguay (Tenti Fanfani, 2005). En casi todas las sociedades modernas se presentan nuevos fenómenos, tales como una tendencia a la incorporación creciente de la mujer en el mercado de trabajo y al mismo tiempo una feminización de las actividades de servicios personales que suponen un trabajo de persona a persona, tales como la enfermería, el cuidado de niños y ancianos, etc. Esta presencia cada vez más masiva de la mujer en los diversos segmentos del mercado de trabajo tiene que ver con un proceso de mayor alcance, que se relaciona con una profunda modificación de los equilibrios de poder entre los géneros a favor de las mujeres.

\section{Cuadro 7 - Distribución de los Egresados de la Carrera de Ciencias de la Educación según el Género, en Porcentajes $(\mathrm{N}=92)$}

\begin{tabular}{|c|c|}
\hline & Género \\
\hline Femenino & 91 \\
\hline Masculino & 9 \\
\hline Total & 100 \\
\hline
\end{tabular}

Fuente: elaboración propia.

\section{La familia}

Los datos sobre el estado civil, la cantidad de hijos, la profesión u ocupación de los padres, el sector educativo de la institución donde asisten los hijos, son características socioeducativas que permiten un acercamiento a las configuraciones familiares de los egresados. En el Cuadro 8 se observa que la mayoría de los egresados son casados o lo fueron, ya que en segundo lugar se ubican quienes están actualmente separados o divorciados, mientras que una menor cantidad de egresados son solteros.

\section{Cuadro 8 - Distribución de Egresados en Ciencias de la Educación de la UNLP entre los Años 1970 a 2009 según Estado Civil, en Porcentajes $(\mathrm{N}=92)$}

\begin{tabular}{|c|c|}
\hline Estado civil & Porcentaje de egresados \\
\hline En unión/casado & 67,39 \\
\hline Separado/divorciado & 17,39 \\
\hline Soltero & 13,04 \\
\hline Viudo & 2,17 \\
\hline Total & 100 \\
\hline
\end{tabular}

Fuente: elaboración propia.

La constitución familiar de los egresados que, en su mayoría decidieron casarse, se completa con los hijos (ver Cuadro 9). En efecto, el 75\% de los egresados tienen hijos y de éstos casi la mitad tienen entre 1 y 2 hijos, 
características que permitiría suponer que los egresados en Ciencias de la Educación presentan una configuración familiar de tipo tradicional y acorde a la clase media, caracterizada por el matrimonio y una cantidad pequeña de hijos.

\section{Cuadro 9 - Distribución de Egresados en Ciencias de la Educación de la UNLP entre los Años 1970 a 2009 según Tengan Hijos, en Porcentajes $(\mathrm{N}=92)$}

\begin{tabular}{|ll|c|}
\hline \multicolumn{2}{|c|}{ Hijos } & Cantidad de egresados \\
\hline SI & & 75 \\
NO & & 25 \\
\hline & Total & 100 \\
\hline
\end{tabular}

Fuente: elaboración propia.

Según lo planteado en el apartado sobre género, la mayoría de los egresados en Ciencias de la Educación son mujeres, esto es, mujeres que trabajan y acorde con ello una cantidad de hijos de entre uno y dos permitiría conjugar la crianza de los hijos y al mismo tiempo llevar a cabo el trabajo fuera del hogar. En relación a la educación de los hijos, en el Cuadro 10 se muestra el sector al que pertenecen las escuelas primarias y secundarias a las que asisten los hijos de los egresados. Estos datos permiten conocer la reproducción de las elecciones educativas a lo largo de las generaciones.

\section{Cuadro 10 - Distribución de Egresados en Ciencias de la Educación de la UNLP según el Sector Educativo que Pertenecen las Instituciones donde Asisten los Hijos, en Porcentajes ( $\mathrm{N}=67$ Egresados)}

\begin{tabular}{|l|c|}
\hline \multicolumn{1}{|c|}{$\begin{array}{c}\text { Sector educativo al que pertenecen las } \\
\text { instituciones }\end{array}$} & $\begin{array}{c}\text { Cantidad de hijos de } \\
\text { egresados }\end{array}$ \\
\hline Instituciones de educación pública & 39,98 \\
\hline Instituciones de educación privada & 24,28 \\
\hline Combinación de instituciones públicas y privadas & 24,28 \\
\hline Hijos aún no escolarizados & 11,42 \\
\hline \multicolumn{1}{|c|}{ Total } & 100 \\
\hline
\end{tabular}

Fuente: elaboración propia.

Una característica entre los egresados y los hijos de éstos es la formación en escuelas primarias y secundarias públicas. La mayoría de los egresados y sus hijos han sido formados en escuelas públicas, y un grupo menor en escuelas correspondientes al sector privado. Respecto de la elección educativa de las familias, Martínez, Villa y Seoane (2009) señalan que dichas elecciones contribuyen a garantizar a las familias una estrategia de reproducción económica, social y cultural. En el proceso de elección se define una trama de relaciones que ordenan las posiciones en el espacio social, tanto de las familias como de las instituciones. En concordancia con 
ello, las características de las instituciones educativas de los egresados tienden a repetirse a lo largo de las generaciones. El 40\% de los egresados envían a sus hijos a escuelas públicas y el $24 \%$ de los egresados combinan escuelas públicas y privadas entre sus hijos. De las escuelas públicas a la que asisten la mayoría de los hijos de egresados, eligen las escuelas normales y los colegios de la universidad. En algunos casos, se combinan los normales con las escuelas básicas o los colegios de la universidad con escuelas básicas.

Para el final de este recorrido por las configuraciones familiares de los egresados, en el Cuadro 11 se muestran las ocupaciones o profesiones de los padres. Con estos datos se busca conocer el perfil laboral familiar a lo largo de las generaciones, como así también las tendencias a repetir una misma ocupación de padres a hijos.

\section{Cuadro 11 - Distribución de los Padres de los Egresados en Ciencias de la Educación de la UNLP según la Profesión u Ocupación, en Porcentajes $(N=92)$}

\begin{tabular}{|l|r|r|}
\hline \multicolumn{1}{|c|}{$\begin{array}{c}\text { Profesión/ocupación de los padres } \\
\text { de los egresados: }\end{array}$} & Padre & Madre \\
\hline Docente & 7,5 & 33,69 \\
\hline Ama de casa & 0 & 33,69 \\
\hline Comerciante & 23,91 & 5,43 \\
\hline Empleado público y privado & 21,73 & 6,52 \\
\hline Oficios & 8,69 & 2,17 \\
\hline Profesional de la salud & 8,69 & 8,69 \\
\hline Ingeniería & 10,86 & 0 \\
\hline Contador, escribano, abogado & 8,69 & 1,08 \\
\hline Productor o empleado rural & 3,26 & 0 \\
\hline Socióloga, Asistente social, psicóloga & 0 & 3,26 \\
\hline N/C & 6,52 & 5,43 \\
\hline \multicolumn{1}{|c|}{ Totales } & 100 & 100 \\
\hline
\end{tabular}

Fuente: elaboración propia.

Los padres de la mayoría de los egresados se dedican a la docencia y tareas domésticas, para el caso de las madres; y al comercio y la administración pública, para el caso de los padres. En menor cantidad, los padres se dedican a las profesiones de la salud, la contabilidad, carreras humanísticas y productores rurales. Es relevante notar que la mayoría de los egresados pertenecen a la ciudad de La Plata (ver Cuadro 14), con lo cual también lo es su familia, siendo la capital de la provincia de Buenos Aires donde se anclan los ministerios estatales, una de las principales actividades de la ciudad junto con el comercio. En este sentido, el Estado como principal empleador no sólo es una característica presente en los cargos de los egresados en Ciencias de la Educación, sino también para el caso de las ocupaciones de sus padres. Para el caso de los empleados o 
productores rurales, se trata de los padres de aquellos graduados que han debido migrar a la ciudad desde el interior para estudiar.

Por otra parte, la docencia es la profesión que más se repite de padres a hijos y por ende, hay una importante reproducción de las elecciones laborales. Al respecto Tenti Fanfani (1995) señala que la docencia es una profesión fuertemente endogámica, que tiende a reproducirse en el interior de las configuraciones familiares: "Cuando la transmisión de ciertas características básicas de un oficio se realiza de una generación a la otra a través de la experiencia relativamente cerrada y privada de la familia, adquiere ciertas formas que la vuelven poderosa" (1995: 67). Así, el espacio tradicional de la docencia se refuerza en el ámbito familiar del graduado y se consolida en el campo laboral.

\section{Las prácticas profesionales}

A partir de 1970, en el campo profesional educativo el desarrollo de la educación no formal ha crecido rápidamente (Coombs, 1970). El concepto de educación no formal destaca la relación e interacción entre los universos de la escuela y de la educación más allá de la escuela y se incluye la totalidad de los estímulos de enseñanza y de aprendizaje existentes en una sociedad. La educación es concebida como una necesidad permanente y como un derecho para todos los individuos y grupos sociales. La educación presenta una mayor flexibilidad, versatilidad y adaptación que la educación formal para atender las diversas necesidades de aprendizaje de cualquier tipo de persona (Sirvent et. al., 2006).

En función de los distintos espacios de inserción profesional en el campo educativo, en el Cuadro 12 se presentan los trabajos actuales de los graduados de las últimas cuatro décadas:

\section{Cuadro 12 - Distribución de Egresados según los Cargos Ocupados al 2012, en Porcentaje $(\mathrm{N}=92)$}

\begin{tabular}{|c|c|c|c|c|}
\hline \multirow[b]{2}{*}{ Trabajo actual: } & \multicolumn{4}{|c|}{ Década de egreso: } \\
\hline & 1970-1979 & 1980-1989 & 1990-1999 & $2000-2009$ \\
\hline Docencia en sistema educativo & 38 & 21 & 15 & 38 \\
\hline $\begin{array}{l}\text { Asesor, consultor, capacitador, gestión en } \\
\text { sistema educativo }\end{array}$ & 62 & 64 & 48 & 33 \\
\hline $\begin{array}{l}\text { Asesor, consultor, capacitador, gestión } \\
\text { en espacios socioeducativos (cárceles, } \\
\text { hospitales, museos, cámaras parlamentarias } \\
\text { y barrios) }\end{array}$ & 0 & 14 & 37 & 29 \\
\hline Totales & 100 & 100 & 100 & 100 \\
\hline
\end{tabular}

Fuente: elaboración propia.

Los egresados durante la década de 1970 tienen una fuerte presencia en los espacios de docencia para la universidad, los institutos de formación docente, las escuelas secundarias y primarias, como así también en gestión y asesoramiento pedagógico en instituciones educativas. Los egresados 
durante la década de 1980, además de trabajar en docencia, gestión y asesoramiento pedagógico en instituciones educativas, también lo hacen en asesoramiento pedagógico en distintos espacios de la sociedad, como es el caso del asesoramiento en la Procuración General de la Corte. También consultoría y capacitación para empresas, organismos estatales, como la Asociación Nacional de la Aviación Civil (ANAC) y no gubernamentales, de talleristas en programas de divulgación de las ciencias y educadores en hospitales. Los egresados durante la década de 1990 mantienen sus trabajos en docencia para el sistema educativo, preferentemente en la universidad y en los institutos de formación docente, junto a la gestión y el asesoramiento, a los que se suman los de gestión de programas estatales, capacitación para el mundo del trabajo, educadores y asesores en museos, en cámaras de legisladores y en cárceles. Para quienes se graduaron entre el 2000 y el 2009, continúan con fuerte presencia en la docencia universitaria e institutos terciarios, como así también en los espacios de asesoramiento pedagógico y curricular para instituciones educativas. A estos espacios se suman los de educador en barrios y evaluador de programas para ministerios.

Asimismo, los egresados combinan casi invariablemente la docencia con actividades de gestión o de asesoramiento pedagógico en distintos ámbitos, sea en el sistema educativo o por fuera de él. La organización del trabajo docente por horas de clases y no por cargos, contribuye a la diversificación de trabajos de los egresados (Terigi, 2008). A diferencia de las escuelas de inicial y primarias, en las instituciones de educación secundaria y terciaria prevalece el currículum mosaico como estructurador del puesto de trabajo: los docentes son designados por horas de clases a dictar. La colección de horas cátedra que es el puesto de trabajo de la mayoría de los profesores dificulta la concentración institucional, pues la unidad de designación no es el puesto sino la asignatura o materia a dictar. La variedad de cargos, prácticas y espacios de inserción de la mayoría de los egresados configura una trayectoria laboral de múltiple y simultánea inserción profesional, donde conviven diferentes instituciones y en la mayoría de los casos también diferentes prácticas como la docencia, la gestión y el asesoramiento al mismo tiempo.

\section{Los trabajos según sector}

Respecto del sector al que pertenecen los cargos de los egresados, los datos del Cuadro 13 advierten que el sector empleador por excelencia de los graduados en Ciencias de la Educación es el Estado.

Según se observa en el Cuadro 13, desde 1970 a 2009 entre el 77\% y el $87 \%$ de los egresados desempeñan trabajos que pertenecen a la esfera pública. Desde la relación entre Estado y los profesionales en Ciencias de la Educación, Novóa (1997) señala que es imposible dilucidar el recorrido histórico de las Ciencias de la Educación sin referirse a los procesos de profesionalización de docentes y de oficialización de la enseñanza. El Estado, a través de los expertos en educación, construyó mecanismos de seguimiento, de evaluación y de regulación basados en categorías creadas 
por los expertos. Los esfuerzos de racionalización de la enseñanza que manifestaron determinadas corrientes de las Ciencias de la Educación son transmisores de una lógica de valorización para consolidar el poder regulador de los expertos en el trabajo de los docentes. Este es el fundamento de un proceso de redefinición de los docentes y de su identidad profesional, para asegurar un nuevo capital simbólico a los especialistas. No obstante, Narodowski (1997) señala que desde 1984 se observa una creciente autonomía y una mayor independencia de estos egresados respecto del poder estatal. La realización periódica de reuniones científicas nacionales e internacionales, la persistencia de varias revistas académicas, la adopción de mecanismos de referato de pares para la admisión de artículos, la consolidación de grupos de investigación en universidades nacionales, la creación de sociedades científicas en materia pedagógica, el fortalecimiento de un mercado editorial de libros pedagógicos en los que se destacan los de autoría de pedagogos argentinos (a diferencia de los años 60 donde primaban los extranjeros). El fortalecimiento de la comunidad académica es la contracara de la tradicional pedagogía de Estado. Se trata de dar respuestas a la diversidad y no apostar al encubrimiento de un proyecto único, hegemónico y totalizador.

Cuadro 13 - Distribución de Egresados según Sector (Público, Privado o Ambos) al que Corresponde/n el/los Cargos Ocupado/s al 2012, en Porcentajes $(\mathrm{N}=92)$

\begin{tabular}{|l|c|c|c|c|}
\hline & \multicolumn{3}{|c|}{ Sector: } \\
\cline { 1 - 4 } Década de egreso: & Público & Privado & $\begin{array}{c}\text { Público y } \\
\text { Privado }\end{array}$ & Totales \\
\hline $1970-1979$ & 76,92 & 7,69 & 15,38 & 100 \\
\hline $1980-1989$ & 78,57 & 3,57 & 17,85 & 100 \\
\hline $1990-1999$ & 74,07 & 7,4 & 18,51 & 100 \\
\hline $2000-2005$ & 87,5 & 0 & 12,5 & 100 \\
\hline
\end{tabular}

Fuente: elaboración propia.

\section{Los lugares de residencia}

El territorio es una construcción social que expresa relaciones entre diferentes sectores (Estado, tercer sector, sector productivo, de servicios) instituciones (municipios, instituciones educativas, hospitales, bancos, etc.) y actores sociales. Constituye un conjunto organizado de actores y recursos que interactúan dialécticamente, una realidad en permanente movimiento de territorialización, donde se conjugan procesos de apropiación y dominio, de subordinación y de resistencias activas que desarrollan los grupos sociales en un contexto determinado (Radonich, Trpin y Vecchia, 2009). De aquí que del Cuadro 14 interesa observar quiénes se movilizaron del interior a la ciudad de la Plata para estudiar y quiénes de los graduados son de La Plata, como así también en qué lugares viven actualmente los graduados de las diferentes décadas. 


\section{Cuadro 14 - Distribución de Egresados en Ciencias de la Educación de la UNLP según los Lugares de Procedencia y los Lugares de Residencia Actuales, en Porcentajes $(\mathrm{N}=92)$}

\begin{tabular}{|c|c|c|c|c|c|c|}
\hline \multicolumn{8}{|c|}{ Lugar de residencia } \\
\hline $\begin{array}{c}\text { Al momento de estudiar la carrera } \\
\text { de Ciencias de la Educación }\end{array}$ & \multicolumn{4}{|c|}{ En la actualidad } \\
\hline Interior & La Plata & Total & Interior & $\begin{array}{l}\text { La Plata Y } \\
\text { alrededores }\end{array}$ & $\begin{array}{l}\text { Grandes ciudades } \\
\text { extranjeras }\end{array}$ & Total \\
\hline 33,69 & 66,30 & 100 & 8,69 & 88,04 & 3,26 & 100 \\
\hline
\end{tabular}

Fuente: elaboración propia.

La mayoría de los egresados en Ciencias de la Educación viven en la ciudad de La Plata. El 66\% tuvo como lugar de residencia la ciudad de La Plata antes de ingresar a la carrera y el 34\% debieron migrar desde el interior (principalmente desde provincia de Buenos Aires) a la cuidad de La Plata para estudiar. La mayor migración se observa desde el interior a La Plata ya que sólo el 12\% reside actualmente en el interior o en ciudades del extranjero, mientras que $88 \%$ reside en la ciudad de La Plata.

Respecto de las posibilidades de inserción laboral en determinados lugares geográficos, la ciudad contiene varias y variadas instituciones donde el egresado puede insertarse en espacios altamente institucionalizados, como la docencia en universidad y en institutos de formación docente. Pero al mismo tiempo, tienen la posibilidad de insertarse en "espacios nuevos" en instituciones no educativas, haciendo referencia a aquellos espacios que no se relacionan con los espacios tradicionales de la profesión, como la educación en cárceles, museos, barrios y programas ministeriales. La cuidad de La Plata cuenta con varios espacios sociales desde donde pensar una propuesta educativa. Cuenta con museos, entre ellos el más importante de la región en el campo de las Ciencias Naturales, el Museo de Ciencias Naturales, el Museo de Arte y Memoria de la Comisión Provincial por la Memoria (CPM) y el Museo de Arte Contemporáneo Latinoamericano (MACLA). Al mismo tiempo, como alberga una gran población en el casco urbano como en los alrededores, cuenta con las cárceles para ambos sexos, tanto de máxima como de mínima seguridad. En el año 2012, la ciudad fue sede del IV encuentro latinoamericano de educadores y tesistas sobre la educación en cárceles. Estas características de la ciudad aportan a la variedad de oportunidades de inserción de los graduados en Ciencias de la Educación.

\section{Conclusiones}

Para Bourdieu [1964] (2009), la definición de la relación que un grupo de actores tiene con sus estudios, se expresa la relación fundamental que su clase social tiene con la sociedad global, el éxito social y la cultura.

En general, los egresados en Ciencias de la Educación provienen de familias de clase sociolaboral media, finalizan la carrera antes de los 25 años 
de edad, son mujeres y la unión o el matrimonio con hijos es la configuración familiar más característica de este grupo de egresados.

En relación a la formación, la mayoría transitaron por escuelas secundarias públicas y la misma característica se reproduce en la formación de sus hijos. En relación al trabajo, desde la década de 1980, el campo profesional de las Ciencias de la Educación se encuentra conformado no sólo por espacios docentes en instituciones educativas, sino en ámbitos y en instituciones que pertenecen al mundo del trabajo o al desarrollo de la ciudadanía en general. Asimismo, la mayoría de los egresados presentan una múltiple y simultánea inserción laboral con predominio del sistema educativo, siendo el Estado el principal empleador.

Finalmente, la mayoría de los egresados residen en lugares donde se podrían construir mayores interrelaciones entre ellos, las instituciones, los espacios de inserción profesional y el acceso a los consumos culturales.

Respecto de los espacios de inserción profesional, existe una ampliación de los marcos de actuación del profesional en Ciencias de la Educación, refieren no sólo a la definición de los caminos de la educación desde la especialización y la investigación, sino también de su concreción a través del asesoramiento, el acompañamiento y la facilitación de condiciones, que desde la educación se realiza, para la mejora de los sujetos o grupos. En este sentido, las prácticas profesionales exceden el espacio de las instituciones escolares y se instalan en diversos espacios comunitarios para atender las problemáticas en el contexto social en el cual emergieron y sobre el cual hay un trabajo educativo que hacer.

\section{Referencias bibliográficas}

BOURDIEU, P. Los herederos: los estudiantes y la cultura. Buenos Aires: Siglo XXI, 2009.

COOMBS, P. H. Nonformal education: myths, realities, and opportunities. Comparative Education Review, v. 20, n. 3, p. 281-293, 1976.

DUBET, F. El declive de la institución: profesiones, sujetos e individuos ante la reforma del Estado. España: Gedisa, 2006.

DUBET, Francois. El declive y las mutaciones de la institución. Revista de Antropología Social, v. 16, p. 39-66, 2007

ECHEVERRÍA, V. El maternaje: ¿espejo de la formación magisterial en México? GénEros, v. 9, n. 26, p. 25-30, 2002.

FERNÁNDEZ, Alfredo. La práctica profesional de la Pedagogía. ANUIES, Revista de la Educación Superior, México, v. 18, n. 72, p. 1-5, 1989. 
FURLÁN, A. La formación del pedagogo: las razones de la institución. In: DEMARCHI, Marta; DUMAR, Diana (Comps.) El campo pedagógico: cuatro visiones latinoamericanas. Montevideo: Educación del Pueblo, 1989.

MARTÍNEZ, A. SOUTHWELL, M. Formación del Estado nacional y constitución de los cuerpos docentes: profesionalización del magisterio en Brasil y Argentina en perspectiva comparada (1820 - 2000). In: VIDAL, Diana; ASCOLANI, Adrián (Coords.). Reformas educativas en Brasil y en Argentina: ensayos de historia comparada de la educación 1820 - 2000. Buenos Aires: Biblos, 2011.

MARTÍNEZ, M. E.; VILLA, A. I. SEOANE, V. Jóvenes, elección escolar $y$ distinción social: investigaciones en Argentina y Brasil. Buenos Aires: Prometeo, 2009.

MARTINIS, P. Las Ciencias de la Educación en Uruguay. Archivos de Ciencias de la Educación, La Plata, n. 5, p. 37-48, 2011.

NARODOWSKI, M. Para volver al Estado: del pedagogo de Estado al pedagogo de la diversidad. Revista Propuesta Educativa, Buenos Aires, n. 17, p. $51-55,1997$.

NÓVOA, A. Professionnalisation des enseignants et Sciences de l'éducation. In: . Histoire \& comparaiso: essais sur l'éducation. Lisboa: Educa, 1998. p. 121 - 127.

POGRÉ, P. Situación de la formación docente inicial y en servicio en Argentina, Chile y Uruguay. Buenos Aires: IESALC/UNESCO, 2004.

RADONICH, M.; TRPIN, V.; VECCHIA, M. T. Movilidad de trabajadores y construcción social del territorio en el Alto Valle de Río Negro. Avá, [Misiones], n. 15, p. 85-102, 2009.

SENNET, R. La cultura del nuevo capitalismo. Barcelona: Editorial Anagrama, 2006.

SIRVENT, M. T. et al. Revisión del concepto de Educación no Formal. Buenos Aires: UBA, 2006.

TENTI FANFANI, E. Una carrera con obstáculos: la profesionalización docente. Revista del Instituto de Investigaciones en Ciencias de la Educación, v. 4, n. 7, p.17-25, 1995.

TENTI FANFANI, E. La condición docente: análisis comparado de la Argentina, Brasil, Perú y Uruguay. Buenos aires: siglo XXI, 2005. 
TENTI FANFANI, E.; GÓMEZ, M. Universidad y profesiones: Miño y Dávila. Buenos Aires, 1990.

WICHNEVSKY, J. Los estudiantes de Ciencias de la Educación de la UBA: las cohortes 1986-2010. ENCUENTRO DE CÁTEDRAS DE PEDAGOGÍA DE UNIVERSIDADES ARGENTINAS, 8., 2011. Anais. .Argentina: La Plata, 2011. p. 1 - 17.

\section{Fuentes}

Anuarios de Estadísticas Universitarias, Secretaría de Políticas Universitarias, Ministerio de Educación de la Nación Argentina.

Anuarios de la Universidad Nacional de La Plata (UNLP), Argentina.

Legajos analíticos de los egresados en Ciencias de la Educación, Oficina de Egresos de la Facultad de Humanidades y Ciencias de la Educación, UNLP, Argentina.

Reglamento y Plan de Estudio de la Facultad de Ciencias de la Educación de la UNLP, año 1915.

María Eugenia Vicente, doctoranda en Ciencias Sociales en la Facultad Latinoamericana de Ciencias Sociales (Flacso) Argentina y docente de Administración en la Educación y en las Instituciones Educativas en Universidad Nacional de la Plata (UNLP), Buenos Aires, Argentina.

eugevicente@yahoo.com.ar

Recibido el 27 de septiembre 2013.

Aprobado el 30 de julio 2014. 